\title{
Pratiques
}

Linguistique, littérature, didactique

141-142 | 2009

La synonymie

\section{Couleur des mots ou synonymie}

\section{Pierre Cadiot}

\section{OpenEdition}

Journals

Édition électronique

URL : https://journals.openedition.org/pratiques/1273

DOI : $10.4000 /$ pratiques. 1273

ISSN : 2425-2042

\section{Éditeur}

Centre de recherche sur les médiations (CREM)

\section{Édition imprimée}

Date de publication : 15 juin 2009

Pagination : 26-38

\section{Référence électronique}

Pierre Cadiot, "Couleur des mots ou synonymie », Pratiques [En ligne], 141-142 | 2009, mis en ligne le 19 juin 2014, consulté le 11 mars 2023. URL : http://journals.openedition.org/pratiques/1273 ; DOI : https://doi.org/10.4000/pratiques.1273 


\title{
Couleur des mots ou synonymie
}

\author{
Pierre Cadiot \\ CORAL, Université d'Orléans
}

\section{Introduction}

On tente dans ce texte d'exposer quelques difficultés générales dont est entachée la notion même de synonymie. Sa mauvaise assise théorique - comme celle de notions connexes telles l'antonymie, l'hyperonymie... voire, bien que très différemment, la polysémie - n'est plus à démontrer, mais un effort systématique d'explicitation d'une telle situation mérite toujours d'être tenté. A quelque niveau qu' on la prenne (langue, dictionnaire, usage, discours, texte, cohérence cognitive, etc.), la synonymie est une notion intuitive, incommode et relative. Aussi les grammaires la définissent-elles en recourant à un critère sémantique vague :

«Les synonymes sont des mots qui, appartenant à la même classe grammaticale, ont à peu près la même signification : dénué, dépourvu, dépouillé, privé. »

«Ordinairement, ce n'est que pour une partie de leurs acceptions que les mots sont synonymes : selon les sens, large a comme synonymes ample, généreux, etc. »(Grevisse, 1986).

La seconde remarque de Grevisse, constatant qu'en pratique, la synonymie ne serait guère autre chose qu'un recouvrement partiel et local, est une sorte de préalable à la question générale. On peut l'illustrer plus complètement avec l'exemple de l'adjectif dur (d'après Le Robert 2005, p. 351). On trouve dans ce dictionnaire la série synonymique suivante (ici simplifiée) :

1.1. - ferme, fort, résistant, rigide, robuste, solide

1.2. - rêche, rude, rugueux

1.3. - coriace, duraille...

2.1. - abrupt, raide, rude

2.2. - ardu, difficile, calé...

2.3. - rigoureux, pénible, sévère...

3.1. - aguerri, courageux, endurant, endurci, stoïque

3.2. - acharné, âpre, farouche, féroce, implacable, sauvage.

4.1. - autoritaire, implacable, inhumain, insensible, sévère...

4.2. - impitoyable, implacable, inébranlable, intransigeant, rigoriste... 
4.3. - turbulent, difficile, dissipé, indiscipliné.

4.4. - blessant, cassant, choquant, cinglant, offensant, rogue, sévère

4.5. - draconien, drastique, rigoureux, strict.

Les critères susceptibles de présider à un tel classement sont nombreux. Par exemple les séries 1 et 2 sont neutres quant à l'opposition humain / non-humain, alors que celles de 3 et 4 accordent une sorte de priorité au trait humain et font ressentir leurs (fréquents) emplois non strictement humains comme dérivés (métonymiques, synecdochiques ou antonomasiques). Un autre critère, celui de la spécificité, peut être retenu pour mettre en évidence le comportement "synonymique " d'adjectifs comme : offensant, draconien, rigoriste, sauvage... qui ne se conçoivent que dans un contexte restreint comme d'éventuels synonymes de $d u r$. On dira très difficilement par exemple d'un problème qu'il est rigoriste!

Les traits « \pm humain » et « \pm spécifique » sont typiquement référentiels : ils marquent les conditions de la fixation d'une extension propre à tel ou tel emploi. On parlera en revanche de traits sémantiques lorsque le supposé synonyme est porteur d'une notion modale explicite alors qu'elle n'est pas explicite dans le mot de référence (ici l'adjectif dur) : il en va ainsi de blessant, cassant, cinglant, rogue, sévère (etc.), où l'on verra plutôt des manières d'être dur ou des modalisations spécifiques sur la notion de dureté. Ou encore, on se rapproche du critère sémantique quand on souligne une variation entre les cas - de loin les plus fréquents - où dur tend à être un hyperonyme pour les adjectifs auxquels il peut être substitué dans un contexte spécifique (draconien, rigoriste...) et ceux où les deux « synonymes » sont plus proches quant à leur généricité, même si le recouvrement n'est bien sûr jamais intégral (rude, difficile).

On prend ainsi une première mesure de la relation générale de synonymie en l'étendant et en l'assouplissant d'emblée par le biais, très provisoire, d'une formule approximative comme :

Pour un contexte donné, dans un domaine de connaissance ou de représentation (plus ou moins) spécifique, pour un certain mode ou une certaine manière d'être, deux mots sont localement substituables, salva veritate.

Il y a donc un sens à explorer deux approches complémentaires de la notion générale de synonymie (la « référentielle " ${ }^{(1)}$ et la « sémantique ») tout en sachant qu'elles ont vocation à se rejoindre, se recouper, voire même à se confondre. Elles fournissent un cadre indispensable pour une éventuelle paramétrisation de la notion.

\section{Référence et sémantique}

Si l'on insiste sur le fait que la synonymie de deux termes ne peut être évaluée que dans les emplois et dans la possibilité de leur substitution (le sens de l'énoncé étant supposé maintenu par la substitution), alors c'est le critère de la référence qui sert de garant.

2.1. Dans un exemple comme Jean craint les orages / Jean redoute les orages ${ }^{(2)}$, les verbes craindre et redouter peuvent être considérés en un sens comme synony-

(1) La notion de synonymie référentielle est explicitement introduite par Klaus Heger (1969). Comme on le verra, la synonymie référentielle est un fait de discours, qui concerne des occurrences et non des types, et qui peut être isolé au titre de condition nécessaire, mais non suffisante, pour tout jugement de synonymie.

(2) Signalé par Vincent Nyckees $(1998,181)$. Les profils syntaxiques différenciateurs sont 
mes, non pas en raison de leur identité sémantique, mais parce que la variation du sens que traduit chaque verbe ne compromet pas (l'identité, ou la quasi-identité, de) la référence. Que redouter exprime une inquiétude relative à un projet particulier (ce qui a un effet d'intensification de l'affect) alors que craindre désigne plutôt un trait comportemental ou psychologique, la nuance n'affecte pas la référence qui, dans le contexte réduit à l'énoncé, est constituée par l'état d'esprit de Jean. Le critère de substitution en contexte est un critère référentiel (ou, rapporté à son corrélat logique, vériconditionnel). Mais, comme nous le détaillerons ci-dessous, la dimension proprement sémantique ne peut à aucun moment être mise hors jeu : si craindre et redouter doivent être distingués comme nous l'avons fait, il reste que leur parenté sémantique est un élément constitutif du jugement de synonymie.

2.2. De même les dictionnaires de synonymes proposent des listes synonymiques comme peur ; crainte ; terreur ; effroi ; stupeur ; étonnement. L'intuition de synonymie est souvent déçue (par exemple par la paire terreur / étonnement). Il vaudrait mieux parler de l'homogénéité - ou de la cohérence - d'un certain champ lexico-sémantique. De multiples critères interviennent pour une dissimilation:

(a) le profilage syntagmatique, ou les possibilités de collocation semi-figée, révèle de grandes différences. Le lecteur le vérifiera aisément en utilisant des tests comme ceux de $u n(N)$, avec $N$, par $N, N$ de, ou encore les collocations verbales (faire $N$, avoir $N$, éprouver $N$, ressentir $N$ ) ; mais aussi bien sûr par l'existence de variantes verbales, etc.;

(b) peur semble renvoyer préférentiellement à un «état», crainte à une « qualité (d'affect) », effroi à une sorte d' " événement » ;

(c) à l'intuition, les catégories très sommaires de (b) peuvent être précisées : peur est replié sur les affects et renvoie à un envahissement de la subjectivité ; crainte se situerait plutôt dans une phase de transfert objectif / subjectif; effroi cantonne la subjectivité dans la perception ; stupeur assume plus encore (que peur) un repli subjectif, mais bien plus ponctuel; étonnement réduit l'affect aux dimensions mêmes de l'événement.

2.3. On peut aussi - parmi mille autres bien sûr - relever un cas comme l'opposition surgir vs. apparaître ( $c f$. « une première difficulté surgit / apparaît alors !»). Comme le note Jean Lecointe (Lecointe, 1993, 27), si surgir et apparaître dans certains contextes sont « référentiellement» substituables, surgir reste attaché à l'idée de passage d'un état à l'autre, désignant très fortement l'origine du mouvement, à partir d'un « point d'ancrage » linguistique initial, qui est peut-être en français à chercher du côté de son doublet sourdre, spécialisé aujourd'hui dans le surgissement de terre d'une eau courante, plutôt que dans le strict sens étymologique latin de "se lever, se réveiller»; apparaître porte très fortement la marque de son " point d'ancrage » dans le registre de la vision, comme paraître ; des difficultés peuvent bien surgir ou apparaître, ce qui revient à peu près au même, les deux verbes n'en conservent pas moins leur spécificité, susceptible de se voir réactivée dans d'autres cas, et toujours infinitésimalement perceptible, à l'état de " coloration », de nuance, "venant fixer la physionomie propre de l'expression choisie, d'une façon qui peut compter dans l'ordre de l'esthétique ou de l'expressivité ». Pour nous, ces dimensions « physionomiques », « esthétiques » ou « expressives » sont au cœur de la sémantique lexicale.

clairs : par exemple, cet orage est redoutable / *craignable. De même, craintif n'a pas de correspondant avec redouter, etc. 
Nécessairement mobilisés conjointement dans toute approche de la synonymie, nous commencerons par rappeler que critère « sémantique » et critère référentiel / (vériconditionnel) s'opposent clairement à un certain niveau. On l'a dit, aucun de ces deux critères considéré à part n'est suffisant pour rendre compte des faits : l'identité ou la parenté des référents visés par deux expressions en contexte n'assure évidemment pas que celles-ci soient substituables l'une à l'autre pour tout contexte. De même la proximité du sens lexical n'entraîne pas eo ipso l'identité de la référence en contexte, qui reste essentielle pour un jugement de synonymie. Les critères sémantique et référentiel n'étant pas réductibles l'un à l'autre, la synonymie impose de distinguer signifié (sémantique) et référence, même s'il s'agit finalement de les relier dans une forme de conditionnement réciproque ${ }^{(3)}$. Il semble en effet que le critère sémantique doive s'appliquer d'abord, le critère référentiel, aussi décisif soit-il pour le jugement de synonymie, ne s'appliquant que dans un second temps. Il serait hors propos d'inférer la synonymie de deux termes à partir du seul fait qu'ils ont la même référence dans un emploi : il est difficile de tenir pour synonymes des expressions comme Fido et ce fidèle quadrupède, bien qu'ils soient (souvent) substituables en contexte pour désigner le chien de mon voisin.

La notion se profile ainsi très généralement selon deux axes : (a.) identité du « référent », (b.) identité du « signifié » :

(a) Exemple rebattu, le vainqueur d'Austerlitz et le vaincu de Waterloo désignent un même individu, 1'empereur Napoléon $1^{\mathrm{er}}$. Des expressions comme rebelles, résistants, terroristes désignent souvent un même référent, mais le font selon des points de vue différents, voire opposés, s'inscrivant directement dans des cohérences discursives et argumentatives.

(b) Des expressions comme citoyen, promeneur, client, parent, voisin... peuvent renvoyer à un même individu (par ailleurs identifiable rigidement par son nom propre), mais elles mettent en jeu des rôles différents pour une identification différente d'un même individu. Comme cela est bien connu, la règle semble être ainsi largement que tout $\mathrm{x}$ est, indépendamment de son devenir prédicatif dans le discours et l'énoncé en cours, identifié dans un réseau a priori, qu'on peut qualifier comme on voudra : jeu de rôles, scénario, script, espace de représentation, voire aussi... point de vue.

(c) Un même référent, ou un même contexte référentiel, connaissent souvent de nombreuses dénominations. Ainsi de ces reliefs routiers qu'à la campagne, on nomme cassis ou dos d'âne, en ville ou en zone péri-urbaine, par exemple ralentisseur (en France) ou coupe-vitesse (en Belgique). Ces expressions ont en commun de désigner (i.e. de permettre la référence à) de courts passages ascendants et descendants sur une route ou une rue et d'appeler à un ralentissement obligatoire de la vitesse du véhicule automobile. Mais plusieurs facteurs brouillent la reconnaissance d'une exacte identité du référent. Cassis et dos d'âne sont des appellations quelque peu archaïques, réservées à des reliefs d'origine naturelle sur les routes de campagne et notamment dans les villages. Ils sont descriptifs, motivés, imagés. Le contraire est vrai de ralentisseur et coupe-vitesse qui sont des dénominations entièrement fonctionnelles et renvoient à un projet non naturel : celui de contraindre légalement les automobilistes au ralentissement dans des zones surtout urbaines. On ne parlera pas dans ce cas de synonymie, en dépit de la parenté des objets désignés.

(3) Dès qu'il y a de la subjectivité, on ne peut plus isoler la référence : cri déchirant, perçant, épouvantable; éclatant. 
2.4. L'identité du « signifié » est plus intuitive. Elle est rarement « absolue », sans doute jamais ! Les situations de synonymie partielle ou approchée, de paraphrase, de «nuance » ou d'équivalence relative sont bien plus fréquentes. On a aussi des variations d'usage (oculiste / ophtalmologiste ; célibataire / non marié $^{(4)}$; bicyclette/vélo; voiture / bagnole), qui ne remettent pas en cause le critère a. Le test suivant est souvent révélateur :
Si Jean est un oculiste, alors il est ophtalmologiste (et réciproquement)
Si ceci est une bicyclette, alors c'est un vélo
? *Si ceci est une voiture, alors c'est une bagnole
* Si Jean est un rebelle (pour moi), il est un résistant (pour toi)
Si Jean est célibataire, alors il est non marié
? Si Jean est non marié, alors il est célibataire.

Cette relation d'implication réciproque, dont les exemples permettent de mesurer la fragilité, se retrouve - variablement - ailleurs dans la langue. Citons notamment, sans du tout approfondir dans ce texte court des questions largement discutées dans la littérature :

- Converses lexicaux : mari / femme ; acheter / vendre ; devant / derrière (mais le livre est sur la table / ? la table est sous le livre ${ }^{(5)}$ );

- Converses syntaxiques : par exemple, actif/passif;

Constitution séquentielle ou globale du mouvement, à la source notamment de la différence entre verbe et nom (Jean part / le départ de Jean ${ }^{(6)}$ )

- Constitution interne des signifiés selon des points de vue différents :

la route monte / la route descend

tiroir / (all.) Schublade [= poussoir]

danger de mort / (it.) pericolo di vita.

De même à un niveau idiomatique :

bureau des objets trouvés / (it.) ufficio oggetti smarriti

(angl.) adults only / (all.) ab 16 Jahre frei.

Ce type d'exemples bilingues illustre aussi à son niveau l'enchevêtrement du référentiel et du sémantique : ils visent une même réalité dans le monde ou dans ses représentations, et d'autre part, ils supposent un apparentement des visées de sens dans les deux expressions, une identité dans la différence, voire même dans 1'opposition. On pourrait dire que, surtout à travers la notion de point de vue, la synonymie crée un fonds sémantique partagé, où on retrouve une forme de valeur de vérité partagée ( si un x est acheté, alors il est vendu; si unx est un tiroir, alors il est aussi un poussoir ; si un objet est trouvé, alors il a été perdu, etc.) autour de laquelle peuvent pivoter des points de vue différents et souvent « opposés » si on les

(4) Cet exemple a été souvent discuté. On rappellera certaines des restrictions à leur équivalence. Le pape est non marié, mais on ne le dit pas «célibataire ». Un enfant de dix ans est (le plus souvent !) non marié, on ne le dira pas non plus « célibataire ». Les usages des mots ne se confondent pas avec la notion. La raison en est que, pour dire que x n' est pas célibataire, il faut qu'il soit attendu ou simplement possible qu'il le soit, donc si l'on veut « pertinent » qu'il ne le soit pas ( $c f$. la notion d'image inverse, Nemo \& Cadiot (1997 a et b)).

(5) Cf. 1'étrangeté, ou 1'inadéquation, de :?? si le livre est sur la table, la table est sous le livre. De même certains types d'emploi plus figurés s'écartent de la reconnaissance d'une conversion sémantique exacte acheter/vendre (ce type est acheté / ce type est vendu). Les sens ne sont plus converses : plus vraiment de synonymie.

(6)

Cf. Claude Vandeloise (2001). 
rapporte à leur seule valeur descriptive, ou plutôt au « scénario » auquel ils sont immédiatement scellés.

\section{Synonymie référentielle?}

On est ainsi poussé à l'hypothèse que la synonymie référentielle n'est qu'un effet qui se stabilise dans la conscience parce qu'a opéré - à cause du caractère en pratique abouti de la référence et de l'impression d'une fixation de valeur de vérité - une forme de neutralisation de toutes les opérations discursives (par exemple tous les faits de thématisation dans le binôme actif / passif, les fonds quasi-idéologiques sous-jacents à une opposition comme (all.) ab 16 Jahre frei / (fr.) interdit au moins de 16 ans, voire argumentatives, pragmatiques, de construction de cette référence.

Pour faire image, on dira encore que dans un cadre frégéen, à cause de la partition stricte en deux couches de sens (sémantique et référence), il est impossible d'expliquer pourquoi l'équation Hesperus $=$ Phosphorus n'est pas triviale, alors que les deux expressions ont le même référent. Pour Frege, il n’y a pas de différence entre :

(1) Hesperus $=$ Hesperus

(2) Hesperus $=$ Phosphorus

puisque, comme en (1), les deux expressions de (2) renvoient à un même référent. Puisque les deux énoncés sont « vrais », le second est aussi peu informatif que le premier. Or - et le moment est venu d'abandonner définitivement Frege ${ }^{(7)}-$ un tel alignement est invalidé par la réalité des discours. Bien qu'également vrais, aucun des deux n'est trivial et tous deux renvoient à des expériences perceptuelles et sémantiques singulières et sont donc porteurs d'indications essentielles. Logiquement tautologique, (1) réécrit en langue (par exemple, Hesperus est Hesperus) peut dans des contextes appropriés prendre différentes valeurs liées au discours : insistance pour qu'on reconnaisse un même objet sous des aspects différents, projection d'un objet identifié sur un objet idéal, décalage temporel entre deux expériences, deux donations que le locuteur propose d'unifier, etc. Quant à (2), permettant l'unification de deux expériences disjointes, il peut aussi viser à en souligner la portée, le caractère inattendu, la nouveauté, etc.

A cet égard, on peut rappeler plus généralement (Cadiot \& Nemo, 1997) 1'existence de nombreux énoncés de facture tautologique (Un sou est un sou; il y a travail et travail ; la loi, c'est la loi), ou quasi-tautologique (Pour un dimanche, c'était un dimanche! Quand je dors, je dors !, Tu veux ou tu veux pas? Je n'ai qu'une parole!, J'étais pas moi-même! Sakai, 2005), paradoxaux (Paris n'est plus Paris,

(7) Dans un cadre frégéen, à cause de la partition stricte en deux couches de sens, il est impossible d'expliquer pourquoi l'équation Hesperus = Phosphorus n'est pas triviale alors que les deux expressions ont le même référent. Et ce y compris en soulignant avec toute l'insistance voulue qu'il y a là deux modes de présentation d'un même objet (ce que fait bien sûr Frege). La raison en est que, pour Frege, l'équation en cause concerne les seuls noms (c'est une question de «mention» et non d' «usage», comme pour les anti-frégéens). Pour Frege, les modes de présentation doivent nécessairement faire partie de la proposition. Il n'y a simplement pas d'autre choix puisqu'ils ne se distinguent pas au niveau des référents. Pour un anti-frégéen au contraire, les modes de présentation doivent être gardés à grande distance du contenu propositionnel. La question de la « référence " sous sa forme frégéenne, exsangue et manichéenne, est loin d'être l'enjeu principal d'une proposition. Les conditions empiriques de donation (et pas seulement de présentation), leur ancrage dans la situation, sont remises au centre du terrain. 
Hesperus est Phosphorus), anomaux (Ce vieil homme est un grand enfant; ce chien est un chat, Cadiot, 2006), d'énoncés métaphoriques (Les femmes sont des mantes religieuses, Ton chien, c'est une vraie poubelle!), ou encore les énoncés de différenciation interne (Il y a vacances et vacances !, C'est pas du travail !). De tels énoncés s'appuient sur une dissociation des occurrences lexicales et à une recharge (modale, évaluative, promotionnelle...) chaque fois singulière des attributs. La position thématique, qu' elle soit explicitement couverte ou non (Je n'ai qu'une parole, c'est pas du travail), étant naturellement spécialisée dans l'identification référentielle quand la position prédicative est une fabrique d'attributions. Ainsi le propre d'énoncés comme un sou est un sou! ou il y a travail et travail! estil de forcer à une dissimilation sémantique, dissimilation qui commence au reste dès la première occurrence, présentée comme ancrage de problématisation, en même temps que comme prise référentielle (la situation à cet égard naturellement dépend du déterminant, mais nous n'en dirons rien ici). On est ainsi engagé dans une sérialisation des valeurs lexico-sémantiques dont l'horizon ultime est aussi ce qu'on appelle "synonymie », étape ultime d'une différenciation qui se marque aussi par des modifieurs épithètes : ce sou (position thématique) qui semble n'être qu'une piètre réalité est aussi une valeur sûre, voire précieuse, être un sou (prédicat), c'est à la fois être peu et être beaucoup. Dans il y a travail et travail, travail peut se traduire indifféremment par " travail intéressant ou (stupide) », « travail utile (ou inutile) », " difficulté », « résultat », " efficacité », " intérêt », " enrichissement », voire " puissance », « énergie », " mérite » [...], travail étant du même coup requis pour faire contraste, à chaque fois différemment, sans plus de précision nécessaire. Parce qu'ils « font» discours, les apparentes tautologies ou quasitautologies voire paradoxes, etc. sont porteurs de sens, peut-être simplement parce qu'ils sont présents et que toute présence doit faire sens ${ }^{(8)}$.

\section{Les mots grammaticaux : prépositions}

La situation la plus fréquente est que, pour deux marqueurs, il existe des contextes où ils paraissent commuter librement (au plan référentiel) sans même parfois qu'une impression sémantique vague fasse ressortir quelque nuance, et que au contraire, dans d'autres contextes, les deux se différencient nettement tant au plan de leur distribution qu'à celui de leur impact sémantique.

A / DE :

(a) aller à / venir de; s'habituer à / se déshabituer de

(b) commencer à / de ; continuer à / de.

Nous avons longuement commenté la différence entre A et DE dans d'autres travaux (Cadiot, 1997). On se contentera ici de rappeler que dans les exemples (a), l'alternance des prépositions se traduit par des valeurs sémantico-référentielles opposées (visée / origine ; engagement / dégagement), alors que dans des exemples comme (b), il n'y a pas de différence de type référentiel, mais une nuance de nature aspectuelle (durativité, habitualité, ponctualité) qui a été décrite par le synonymiste Pierre-Benjamin Lafaye : on commence, disait-il à peu près, à faire une action ou une suite d'actions qui n'est pas refermée dans des limites précises,

(8) Les travaux de Anna Wierzbicka (1987) et de Tomohiro Sakaï (2005), notamment, tendent à montrer que ces figures discursives sont largement différentes d'une langue à l'autre. 
qu'on continuera ou qui se continuera indéfiniment. On commence de faire une action unique, qui constitue une œuvre fixe... qui s'achève en plus ou moins de temps, qui a un commencement, un milieu et une fin. Un enfant commence à parler, un orateur commence de parler (P.-B. Lafaye, 1861, Dictionnaire des synonymes de la langue française). A et DE sont ainsi des antonymes dans certains contextes et de quasi-synonymes dans d'autres. La différence entre synonymie référentielle et synonymie sémantique (qui peut rejoindre l'intuition référentielle d'antonymie) est précieuse dans ce cas.

Une situation comparable se retrouve dans l'alternance POUR/CONTRE (Cadiot 1991) :

\section{POUR / CONTRE :}

(a) voter pour / contre

(b) médicaments pour / contre la grippe.

L'antonymie référentielle est évidente dans (a), mais elle tient à l'adressage identique et à la constitution d'une alternance matérialisée dans la syntaxe. En (b) par contre, deux phases de constitution de l'objet apparaissent nettement: celle où les médicaments sont à propos de / destinés à " la grippe " (d'où la préposition pour), et celle, plus interne au mot, où le médicament a une valeur plus processuelle de lutte (d'où la préposition contre).

Plus généralement, le choix prépositionnel exprime un modalité de constitution thématique, parfois bien catégorisé cognitivement (dans / en / avec / par / au (le) train), parfois en deçà d'une catégorisation objective, proto-sémantique, ou thématisable au niveau de rôles ou fonctions sémantiques intuitivement plus vagues : aspect, existentiel / locatif, etc.

J'ai poussé l'analyse jusqu'à reconnaître que la signification n'était rien d'autre qu'une question de point de vue, de modalité de constitution de l'expérience. Ceci ressort avec une particulière clarté des noms communs, à condition que 1'on prenne par principe en compte l'ensemble de leurs emplois, d'où les immenses progrès rendus possibles par les nouvelles facilités qu'offrent les techniques documentaires : pas de barrière entre littéral et figuré (innere Sprachform) : la métaphoricité est une fonction de l'usage ; sa valeur ne se fait ressentir comme telle que parce qu'elle est indexée sur un contexte spécifique.

\section{Indétermination de la notion : idée principale vs idée accessoire}

Gabriel Girard, dans La justesse de la langue française ou les différentes significations des mots qui passent pour synonymes, (1718:115) écrit:

«...les synonymes représentent tous une même idée principale, et [...] ils ne diffèrent que par des idées accessoires et particulières ».

Nicolas Beauzée, dans la Préface à son édition des Synonymes français de Roubaud $(1771: 7)$ écrit à son tour :

"Quand on ne considère dans les mots de même espèce qui désignent une même idée principale, que cette idée principale et commune, ils sont synonymes, parce que ce sont différents signes de la même idée ; mais ils cessent de l'être, quand on fait attention aux idées accessoires qui les différencient...».

Or il nous semble que cette opposition idée principale et commune vs idée accessoire et particulière se distingue mal de la polysémie radiale ou de l'hyperony- 
mie, risquant ainsi de brouiller les frontières entre les catégories du « structuralisme » sémantique. Voyons l'exemple du verbe habiller, en nous appuyant sur une analyse de Walther von Wartburg (1969) :

Habiller un arbre : apprêter

Habiller un cheval : harnacher, mais aussi castrer, étriper

Habiller une armée : équiper ${ }^{(9)}$.

(9) D'après Walther von Wartburg: « Le verbe habiller signifiait en vieux français "arranger pour un certain usage", c'est-à-dire qu'il appartenait à la langue générale. Toutefois, il se scinda bientôt en un grand nombre de significations spéciales. Les jardiniers l'emploient dans le sens d' "apprêter un arbre qui doit être transplanté", alors qu'il veut dire pour le paysan "nettoyer une prairie au printemps de toutes les ordures qui peuvent l'encombrer", ou encore "carder le chanvre une première fois"; dans la bouche du charretier, il signifie "harnacher le cheval", dans celle du boucher "étriper", dans celle du vétérinaire "castrer", dans celle du tanneur "préparer la peau qui doit être exposée à la flamme"; quand le mécanicien prononce ce verbe, il désigne par là l'assemblage correct de tous les rouages et balanciers de la montre ; le meunier y associait l'idée de recouvrir de toile les ailes du moulin; pour le batelier, habiller voulait dire "accoupler correctement" un radeau ; le potier enfin entendait l'adjonction d'anses et de pieds aux ustensiles qu'il fabriquait et qui marquaient le couronnement de son œuvre. Le verbe avait donc littéralement éclaté au point de vue sémantique dans une série de significations spéciales, que nul n'aurait pu comprendre en dehors du cadre de son activité ; il vivait dans un grand nombre de langues professionnelles mais fort peu dans la langue commune. Or il existait encore une autre signification spéciale que nous n'avons pas mentionnée : c'était "équiper pour le combat". Ce sens donne naissance vers 1400 à la signification général de "vêtir" et c'est dans cette signification que le verbe passe dans la langue générale où il acquiert rapidement droit de cité. Il a rompu par là sur un point ses chaînes étroites et s'élève désormais librement. On croirait voir une branche maîtresse qui jaillit soudain d'un buisson et domine bientôt de son ombre l'amas des modestes rameaux. » (von Wartburg, 1969, 169).

Citons aussi le cas de train (d'après Le Robert, D.H.L.F, $1992: 2148$ ) : «Un mot comme "train" a un spectre potentiel d'application référentielle qu'il doit, comme tant d'autres, à son origine dans une notion qui implique l'expérience générale : si "train" a d'abord désigné (v. 1160) un ensemble de choses, et dans les chansons de geste, de cadavres dont la terre est jonchée, ses autres sens anciens (convoi de bêtes voyageant ensemble (v. 1190), file de bêtes de somme voyageant ensemble avec le personnel de service (v. 1240) et "ensemble de domestiques, de chevaux, de voitures accompagnant une personne" (1240, trahin) (2148) [...]».

S'il « met (ainsi) l'accent sur l'idée de file, de suite en mouvement... amorçant plusieurs sens modernes » n'est-il pas en fait évident qu'une telle idée sur laquelle les usages, anciens et modernes, «mettent l'accent » est le seul candidat raisonnable pour la signification lexicale, une fois qu'on l'a donc ainsi clairement distingué de la désignation. Plus peut-être que sur l'idée de "suite en mouvement », il faudrait insister sur la notion associée ou complémentaire de «station». On citera les exemples :

- File de bêtes de somme ou du personnel de service qui suivent qn.; un train de chevaux,

- 1660 : train de bateaux, train de chariots, train de laminoir, train de vagues, train d'ondes

$-19^{\mathrm{e}}$ siècle : soldat du train, train d'artillerie,

- Enchaînement des choses : un train de pensées.

Soit encore :

- « Nous voici dans un vilain train de neiges, de pluies et de vents terribles » (Mme de Sévigné)

- train de maison $=$ domesticité

- bruit, tapage : " la salle s'emplissait de train, de rire » (A. Daudet).

Ce n'est que depuis 1840 qu'est apparu le sens « chariots tirés par une locomotive».

- allure (pour les chevaux) : aller petit train, grand train, à fond de train, un train d'enfer

litt. marche : «voilà le train du monde » (Montaigne, 1580). Laisser le monde aller son train (train-train)

- conduite, « en vain de son train ordinaire, on veut le désaccoutumer» (La Fontaine) 
- Soit, habiller est un synonyme de apprêter, harnacher, équiper (qui sont censés apporter une «idée accessoire »).

— Soit, c'est un hyperonyme, engageant en aval un mécanisme d'instanciation.

- Soit, c'est un polysème : les trois valeurs d'habiller sont structurées radialement (arbre, cheval, armée). On parlerait alors d'un mécanisme de particularisation.

La notion d' "idée accessoire » reste des plus ambiguës : on peut rappeler que si connotation est, en langue ordinaire et en sémiologie peut-être, de l'ordre de l'accessoire ou de l'adventice, elle renvoie aussi (comme l'a montré par exemple un John Stuart Mill) au contenu conceptuel ou notionnel.

Le synonymiste Roubaud au $18^{\mathrm{e}}$ siècle considérait que les mots (les polysèmes) étaient dotés d'un principe de motivation issu de leur forme, qui à travers tous les usages, révélait une « unité des sensations et des affects ». Chaque mot est assimilable à un « schème très général évoquant un modèle perceptuel reconnu indifféremment dans une réponse des organes de la perception, dans une sensation interne, dans une relation à l'espace et par l'espace, au temps » (Berlan, $2005: 83$ ). Ou encore, « [Roubaud] postule une sorte d'épaisseur mémorielle des mots qui ne peuvent que persévérer dans leur être à travers les extensions de sens, selon des possibilités ou des impossibilités liées au cœur d'un sémantisme positif et proprement singulier » (Berlan, 2005:88).

\section{Conclusion}

Il n'y a pas de synonymie au sens fort, parce que ce qu'on appelle référence, c'est, plutôt que des individus isolés, des situations, des événements... voire des impressions ${ }^{(10)}$. Ces limites à l'analyticité et à l'univocité référentielles, reconnues par les psychologues eux-mêmes ${ }^{(11)}$ et que cherchent à capter des notions

- éléments de train de vie

- « en train » : personne ne se sentait en train, ni le maître ni les élèves

- « en train de »

- partie qui traine : avant-train, arrière-train

pop. : le derrière : filer le train à qn.

tech. : partie d'une presse d'imprimerie / mise en train: réglage de la pression sur les divers éléments de la forme.

(10) La caractérisation habituelle des noms en termes de domaines de représentation d' "objets » (lieux, choses, ensembles) [...] de dénotés catégoriels procède d' une confusion avec le syntagme. La question générale de la synonymie, parasynonymie, etc. peut reprendre des couleurs si on revoit les noms eux-mêmes comme des accès, des indications, des modalités de donation, etc. des index de valeur dans le champ de la perception et de la sensibilité.

(11) Soit par exemple cette citation de Jean-François Le Ny : «On ne dispose pas aujourd'hui de donnée neurobiologique qui nous informe de façon précise et fiable sur ce qu'est le substrat cérébral d'un concept spécifique. Il se pourrait bien même que la rechercher ne nous fournisse jamais de telles preuves neurobiologiques directes, c'est-à-dire qui puissent nous apprendre seules, en l'absence de données comportementales et cognitives associées, ce qui fait que telle activité cérébrale est le substrat d'une représentation et d'un concept, celui de $<$ jacinthe $>$ par exemple [...]. Ce qui est l'essentiel d'un concept, c'est la relation qui reste objet de débat et de recherché, entre le concept et ce dont il est le concept, sa référence. La spécificité du concept de $<$ jacinthe $>$, c'est d'exister “à propos" des jacinthes, d'entretenir une relation avec les jacinthes. On aimerait que cette relation soit bien déterminée, mais elle ne peut l'être, parce que ce à quoi le concept de $<$ jacinthe $>$ est relié, c'est à des perceptions de jacinthes, ou à des phrases ou des pensées concernant les jacinthes. Le substrat 
comme celles d'holisme, de sous-détermination constitutive, de variabilité des modalités d'accès (etc.) sont au cœur de toute théorie de la synonymie. C'est pourquoi nous défendons depuis longtemps l'idée que des propriétés qui n'auraient pas de corrélat intentionnel dans le monde des pratiques et des expériences n'ont aucune place en sémantique lexicale. Les mots doivent d'abord être vus comme des index de discours, donc dans leur texture locale. Les motifs lexicaux (Cadiot \& Visetti, 2001) ne sont que des supports d'élaboration pour des opérations de profilage (leur mise en syntagme, en phrase, en texte, leurs collocations ou figements) et de thématisation (leur vocation à parler du monde, leur dimension intentionnelle, ou, si l'on veut, référentielle). Ils ne se stabilisent que par l'entremise d'opérations textuelles dont une cristallisation sensible est l'ensemble des effets de figement, semi-figement, délocutivité formulaire (imposer silence, faire confiance, prendre congé (faire ses adieux) / prendre un congé (prendre des vacances) (Anscombre, 1982). Ou encore dans le cadre de collocations privilégiées, qui donnent un meilleur accès aux motifs eux-mêmes :

Louche : servir à la louche / une louche de compliments

Table : dresser / disposer / passer à / servir à... table

Euf: sortir de l'œuf/ tuer dans l'œuf, crâne d'œuf(Visetti \& Cadiot, 2006 : 49).

Contrairement à une certaine vulgate bottom / up, que l'on trouve clairement dans la notion de compositionnalité, la mise en syntagme n'est jamais une simple instanciation « libre » et nous renvoie donc à cette notion de texture locale ${ }^{(12)}$. Il n'y a donc pas de référence indépendante des situations et des textes, donc pas de synonymie : les mots sont en inter-constitution et en inter-définition syntagmatique, dans des réseaux enchevêtrés multiples. Les petits paradigmes du « structuralisme » (déjà présents chez Michel Bréal et d'autres) sont des méta-opérations chirurgicales, dépendantes de l'approche dictionnairique. On le sait, mais on ne tire pas suffisamment cette conséquence inévitable que l'idée que le discours est une mise en œuvre de la langue est une contradiction dans les termes.

Pourquoi est-il sur le fond, à la limite, sauf par commodité, impossible de parler de synonymie ? Parce que la notion même isole le mot (ou le morphème) en y voyant une dénomination et / ou une catégorie référentielle : elle coupe ainsi le mot de sa forme, de sa motivation et de sa genèse internes, en même temps que de son devenir discursif.

Or nous rappellerons que pour nous le mot est avant tout une modalité d'accès et de constitution, c'est-à-dire :

neuronal correspondant, qu'on ne peut caractériser autrement que comme "la population des neurones qui sont actives chez un individu quand il perçoit des jacinthes, parle de jacinthes, ou pense à des jacinthes" comporte vraisemblablement une part notable qui est anatomiquement différente entre les individus, et même aléatoire » (Le Ny, 2004).

(12) On souscrit pour l'essentiel à cette analyse de Jean Lecointe : "Pour reprendre une notion mathématique, due à René Thom, dont nous usons peut-être ici imprudemment, mais dont l'application en linguistique nous paraît profitable, nous pourrions dire de la synonymie, et au-delà du choix de l'expression linguistique, qu'il s'agit, aussi bien historiquement, collectivement, qu'individuellement, d'une situation de catastrophe. Autrement dit, une situation limite, telle que plusieurs structures sont susceptibles de s'y appliquer avec plus ou moins de convenance, et où, en fin de compte, il suffit d'un impondérable pour tout faire basculer d'un côté plutôt que de l'autre. Un peu comme une ligne de partage des eaux sur un bombement assez aplati : il existe des zones du bombement où il fait peu de doutes que la goutte de pluie dévalera plutôt un versant que l'autre, mais il existe également une zone indécise, sur le replat» (Lecointe, $1993: 25$ ). 
1. une Gestalt combinant à des degrés variables forme / praxéologie / axiologie ;

2. il est syntagmatisé avec des collocations préférentielles, ou semi-figements, ou encore une syntaxe formulaire ${ }^{(13)}$;

3. il réfère de manière chaque fois singulière.

Ces mots différents qui atteignent un même "référent ", ils le font de manière fortement aléatoire parce qu'ils sont associés à des programmes de sens qui les dépassent, ou encore à des horizons thématiques différents ${ }^{(14)}$. Pour parler de synonymie (de toute façon, "faible »), il faut donc commencer par identifier ces programmes de sens en cours de déploiement, ce qui comporte avant tout de ne pas voir dans le mot un signe isolé, refermé sur une fonction purement dénominative et catégorielle, de ne pas non plus chercher à l'identifier avec une valeur de base, ou prototypique. La référence doit être revue fondamentalement comme un effet des discours et la sémantique réorientée vers les modalités de construction du sens et de la valeur.

\section{Références bibliographiques}

ANSCOMBRE, J.-C. (1982) : «Un essai de caractérisation de certaines locutions verbales », Recherches linguistiques, Université Paris $8: 5-37$.

BERLAN, F. (2005) : «Un synonymiste censuré : Roubaud ou le rôle du mot-objet en synonymie distinctive », in La langue, le style, le sens, Etudes offertes à AnneMarie Garagnon, Ed. L'improviste : 81-91.

CADIOT, P. (1991) : De la grammaire à la cognition, la préposition pour, Paris, Editions du C.N.R.S.

- (1997) : Les prépositions abstraites en français, Paris, A. Colin.

- (2006) : «Métaphore prédicative nominale : genèse de la constitution de l'objet dans le champ subjectif », in Aux carrefours du sens, hommages offerts à Georges Kleiber pour son $60^{e}$ anniversaire, Leuven, Paris, Dudley, Peeters.

CAdiot, P. \& Visetti, Y.-M. (2001) : Pour une théorie des formes sémantiques. Motifs, profils, thèmes, Paris, P.U.F..

CAdiot, P. \& Nemo, F. (1997) : «Analytique des doubles caractérisations », Sémiotiques, $13: 23-54$.

(13) Ainsi du mot carton, d'où vient ça cartonne! Par le biais de nombreuses emplois dits dérivés, idiomatiques ou formulaires : «ces crimes de carton » (S. de Beauvoir), «taper le carton », «aller au carton », «faire un carton », où la valeur de base de carton(ner) s'apparente à un motif composite où interviennent les notions de heurt, de front, de fragilité, d'apparence trompeuse, d'illusion... toujours plus ou moins renouvelées par des thématiques spécifiques : la matière carton, le jeu, les engagements.

(14) Point depuis longtemps reconnu dans la tradition humboldtienne, notamment russe. Ainsi de A. Simvol Potebnja, présenté par Jacqueline Fontaine : «Le mot n'est pas 1'expression d'une pensée toute faite [...] Au contraire, il l'est seulement dans la mesure où il est le moyen pour créer cette pensée ; la forme intérieure, contenu objectif unique [edinstvennoe] $\mathrm{du}$ mot, n'a de signification que parce qu'elle change d'apparence [vidoizmenjat'] et qu'elle réalise au mieux les assemblages de perceptions qu'elle trouve dans 1'âme » (Fontaine 2006 : 55). Dans l'ordre de la langue, tout se passe comme si la forme intérieure avait une fonction de mise en route de la langue, lui imprimant le mouvement comme si elle s'effaçait ensuite pour confier la langue à l'aventure des actes de discours (ibidem, 58). 
FOnTAINE, J. (2006) : " La “innere Form" : de Potebnja aux formalistes », in « L'Allemagne des linguistes russes », Revue Germanique Internationale 3 : 51-62.

GIRARD, G. (1718) : La justesse de la langue française ou les différentes significations des mots qui passent pour synonymes, Texte établi par M.G. Adamo, 1999, Fasano-Paris, Schena-Didier Erudition.

Grevisse, M. (1986), Le Bon Usage, Gembloux, Duculot.

DICTIONNAIRE DES SYNONYMES ET NUANCES (2005) : Paris, Le Robert.

HEGER, K. (1969) : «L'analyse sémantique du signe linguistique », Langue Française, $\mathrm{n}^{\circ} 4$, Larousse : 44-66.

LAFAYE, P.-B. (1861) : Dictionnaire des synonymes de la langue française, Paris, Hachette.

LeCOINTE, J. (1993) : Dictionnaire des synonymes et des équivalences, Paris, Le livre de poche.

LE NY, J.-F. (2004) : Comment l'esprit produit du sens, Paris, Editions Odile Jacob.

LE ROBERT (1993) : Dictionnaire Historique de la langue française, Paris, Dictionnaires Le Robert.

NEMO, F. \& CADiOT, P. (1997a et b) : «Un problème insoluble ? », Revue de Sémantique et Pragmatique $\mathrm{n}^{\circ} 1$ et 2 : Presses de 1'Université d'Orléans.

NyCKEES, V. (1998) : La sémantique, Paris, Belin.

RouBAUD, Abbé (1796) : Nouveaux synonymes français, nouvelle édition, Paris (4 volumes).

SAKAI, T. (2005) : «Etre soi-même : étude contrastive du français et du japonais », BULA G. Divergences dans la traduction entre les langues orientales et le français ; $\mathrm{n}^{\circ}$ 30, Presses Universitaires de Franche-Comté : 147-164.

VANDELOISE, C. (2001) : Aristote et le lexique de l'espace, rencontres entre la physique grecque et la linguistique cognitive, Editions CSLI, Université de Stanford, Stanford.

VISETTI, Y.-M. \& CADIOT, P. (2006) : Motifs et proverbes, Essai de sémantique proverbiale, Paris, Presses Universitaires de France.

W ARtBurg, W. (VON) (1969) : Problèmes et méthodes de la linguistique, Paris, Presses Universitaires de France.

WiERZBICKA, A. (1987) : « Boys will be boys : "radical semantics" vs. "radical pragmatics" », Language 63. 1 : 95-114. 